\title{
Autoimagem de pacientes com colostomia
}

\section{Self-image of colostomy patients}

Autoimagen de los pacientes con colostomía

Ana Fátima Souza Melo de Andrade

ORCID: https://orcid.org/0000-0002-7024-6175

Centro Universitário Estácio de Sergipe, Brasil

E-mail: anafatimamelo@hotmail.com

Juliana Cabral Azevedo

ORCID: https://orcid.org/0000-0001-8228-434X

Centro Universitário Estácio de Sergipe, Brasil

E-mail: juenfa.14@gmail.com

Weber de Santana Teles

ORCID: https://orcid.org/0000-0003-1770-8278

Centro de Hemoterapia de Sergipe, Brasil

E-mail: arteecura@hotmail.com

Alejandra Debbo

ORCID: https://orcid.org/0000-0002-7743-5921

Universidade Tiradentes, Brasil

E-mail: aledebbo@hotmail.com

Max Cruz da Silva

ORCID: https://orcid.org/0000-0002-6944-5986

Faculdade Pio Décimo, Brasil

E-mail: maxlfi@hotmail.com

Ruth Cristini Torres

ORCID: https://orcid.org/0000-0002-8664-192X Instituto de Hematologia e Hemoterapia de Sergipe, Brasil

E-mail: ruthcristini@gmail.com

Marcel Vinícius Cunha Azevedo

ORCID: https://orcid.org/0000-0002-5312-3333

Centro Universitário Estácio Sergipe, Brasil

E-mail: marcelvinicius49@gmail.com

Ângela Maria Melo Sá Barros

ORCID: https://orcid.org/0000-0003-4087-3247

Universidade Federal do Rio de Janeiro, Brasil E-mail: angelsamelo@hotmail.com

Taíssa Alice Soledade Calasans

ORCID: https://orcid.org/0000-0003-0460-4437

Universidade Tiradentes, Brasil

E-mail: taissa.asc@gmail.com

Maria Hozana Santos Silva

ORCID: https://orcid.org/0000-0001-5742-5366

Faculdade Ages de Medicina, Brasil

E-mail: hosana p@hotmail.com

Paulo Celso Curvelo Santos Junior

ORCID: https://orcid.org/0000-0001-5834-6782

Universidade Tiradentes, Brasil

E-mail: paulo.curvelo.jr@gmail.com

\section{Resumo}

Este estudo tem como objetivo avaliar a autoimagem dos pacientes ostomizados. Trata-se de uma pesquisa bibliográfica, no qual obteve-se embasamento em artigos sobre o presente dos últimos dez anos. O trabalho dos enfermeiros é fundamental para o gerenciamento dos cuidados dos pacientes ostomizados. E o enfermeiro deve estar preparado para prestar todas as informaçõescom clareza, cumprindo determinadas etapas, tais como: a internação, exames préoperatório, o trans operatório, o pós operatório, finalizando com a sua alta hospitalar. No cuidado aos pacientes portadores de ostomias intestinais conclui-se noperíodo pós-operatório, a ocorrência de dificuldades em aceitar a nova condição deostomizado, e o déficit de conhecimentos sobre o cuidado com o auto cuidado. Então,surge a necessidade da consulta de enfermagem no pré operatório de ostomias, nosentido de: possibilitar ao paciente melhor aceitação da ostomia e do seu tratamento;estar orientando o ostomizado e seus familiares quanto aos cuidados, associações egrupos de apoio.

Palavras-chave: Colostomia; Autocuidado; Adaptação psicológica. 


\begin{abstract}
This study aims to assess the self-image of ostomized patients. This is a bibliographic research, in which articles based on the present of the last five years were obtained. Nurses' work is fundamental for the management of care for ostomized patients. And the nurse must be prepared to provide all the information clearly, fulfilling certain steps, such as: hospitalization, preoperative exams, the transoperative, the post operative, ending with his discharge from hospital. In the care ofpatients with intestinal ostomies, it is concluded in the postoperative period, the occurrence of difficulties in accepting the new condition of ostomates, and the lack of knowledge about self-care. Then, there is a need for nursing consultation in the preoperative ostomy, in the sense of: enabling the patient to better accept the ostomy and its treatment; to be guiding the ostomate and his family regarding care, associations and support groups.
\end{abstract}

Keywords: Colostomy; Self-care; Psychological adaptation.

\title{
Resumen
}

Este estudio tiene como objetivo evaluar la autoimagen de los pacientes ostomizados. Se trata de una investigación bibliográfica, que se basó en artículos sobre el presente de los últimos diez años. El trabajo de las enfermeras es fundamental para gestionar el cuidado de los pacientes ostomizados. Y la enfermera debe estar preparada para brindar toda la información de manera clara, cumpliendo con ciertos pasos, tales como: hospitalización, exámenes preoperatorios, transoperatorios, posoperatorios, finalizando con el alta hospitalaria. En la atención de pacientes con ostomías intestinales, se concluye en el postoperatorio, la aparición de dificultades para aceptar la nueva condición de los pacientes con ostomías y el desconocimiento sobre el autocuidado. Por tanto, es necesaria la consulta de enfermería en el período preoperatorio de las ostomías, con el fin de: permitir que el paciente acepte mejor la ostomía y su tratamiento; estar asesorando a los ostomizados y sus familias sobre cuidados, asociaciones y grupos de apoyo.

Palabras clave: Colostomía; Autocuidado; Adaptación psicológica.

\section{Introdução}

As doenças relacionadas ao trato gastrointestinal na maioria das vezes levam as pessoas a ficarem com colostomia/ileostomia, que podem ser temporárias, paracicatrização e outras são definitivas. Os indivíduos que passam por estes procedimentos cirúrgicos, ficam com trânsito intestinal desviado, com a autoestimae a autoimagem abalada e em muitos casos, estas pessoas se privam da vida socialpreferindo se resguardar devido ao uso da bolsa de colostomia (Ricardo, Santos \& Palermo, 2018).

Com essas alterações físicas e o psicológico abalado, o apoio familiar é muito importante para a recuperação dos ostomizados, uma vez que diversas ações do cotidiano são importantes para possibilitar uma boa recuperação e facilitação no processo de reaprendizagem de várias atividades do cotidiano, a exemplo de posições para dormir, ajustes de roupas no corpo e até mesmo, o redescobrimentosexual (Ferreira, 2017).

A inserção do paciente na sociedade comapoio familiar contribui para sua autoestima, um ponto importante para sua recuperação, pois é através deste suporte que o indivíduo terá sua mentalidade trabalhada para compreender a sua nova realidade do cotidiano com os estomas (Batista, 2011).

Na década de 1950, ocorreram também as preocupações com o cuidado direcionado ao ostomizado, surgindo as Associações de Ostomizados, atividade está reconhecida como Estomaterapia. O mesmo Turnbull conseguiu enxergar o ostomizado, além da técnica cirúrgica, identificava-o como um ser humano que necessita de instruções e autocuidado, motivando-o ao convidar para desenvolver um trabalho de orientação aos ostomizados, com uma de suas clientes, Norma Gill, ileostomizada após uma retocolite ulcerativa, que demonstrava interesse em ajudaroutros ostomizados em suas necessidades (Cesaretti, 2014).

Os cuidados de manipulação e higienização com os estomas são importantespara evitar infecção, a compreensão de atos como se trocar uma bolsa de colostomia, observar a pele e interpretar caso tenha alguma alteração e saber lidarcom isto, são de grande necessidade. Esses cuidados começam desde a realizaçãodo procedimento cirúrgico até sua alta e a enfermagem tem o papel de orientar o paciente no pós-operatório e com os cuidados necessários (Ferreira, 2017).

Vale ressaltar que ainda no pré-operatório, é importante que a equipe médicaouça o paciente, avaliar seu psicológico e compreender seus sentimentos de medoe ansiedade e assim explicar toda informação necessária para seu paciente, informando 
todo o procedimento cirúrgico, a começar pela anestesia (Ferreira, 2017).

No hospital que será realizado o procedimento, é crucial a equipe passar confiança, mas explicar que podem acontecer algumas intercorrências, uma vez que nem sempre o paciente está preparado para sair ostomizado de uma cirurgia, com isso o psicológico pode ser afetado (Ferreira, 2017).

Em suma, nosso modo de viver atualmente, onde temos alta exposição a alimentos industrializados e um estilo de vida sedentário, acabam por prejudicar nossa saúde e 5 aumentam a chance de acometimento de doenças que levem a necessidade da ostomia , desta forma, é importante compreender como o pacientelida com o medo e ansiedade no pré-operatório, mas também como ele se reconectaconsigo mesmo após a cirurgia (Batista, 2011).

O presente trabalho apresenta que os pacientes ostomizados são acometidos por severas mudanças em sua imagem corporal, influenciando futuramente, seus sentimentos resultantes serão os mais diversos.

Portanto justifica-se pela necessidade de extrema relevância ao atendimentoprestado pelo profissional de enfermagem. Tendo como alicerce o fornecimento de informações que sirvam de auxílio na sua adaptação à nova condição de vida, estimulando para que ele realize o auto cuidado, ser o elo entre os familiares e o ostomizado, para que a reabilitação seja facilitada. Este estudo tem como objetivo avaliar a autoimagem dos pacientes ostomizados

\section{Metodologia}

O presente estudo trata-se de uma revisão integrativa da literatura, pertinenteao tema, abrangendo e condensando o conhecimento científico já desenvolvido sobre a temática investigada. Uma revisão integrativa de caráter qualitativo que busca atingir o objetivo geral da pesquisa. Constituída das seguintes etapas: identificação do tema e do problema da pesquisa; pesquisa dos descritores; estabelecimento dos critérios de inclusão e exclusão de artigos; definição das informações a serem extraídas dos estudos; análise sistemática dos dados; interpretação dos resultados e síntese dos dados. A revisão integrativa de caráter qualitativo estabelece o conhecimento atual referente a uma temática específica, pois investiga e condensa os resultados sobre o mesmo tema, compreendendo e explorando a temática abordada analisando as suas semelhanças e peculiaridades.(Mendes, 2010).

A busca das fontes foi desenvolvida na base de dados da Biblioteca Virtual em Saúde (BVS), Base de Dados em Enfermagem (BDENF), Literatura Latino- Americana e do Caribe em Ciências da Saúde (LILACS), e na biblioteca eletrônica Scientific Electronic Library Online (SciELO). Utilizando os descritores: Colostomia, Autocuidado e Adaptação psicológica. Os critérios de inclusão foram: ano de publicação, últimos dez anos, 2010 a 2020, idioma em português, texto completo, que abordasse o tema proposto. E os critérios de exclusão foram artigos que não foram publicados na integra, como teses, dissertações e editoriais.

Este estudo tem como relevância acadêmico e social, o intuito de informar oleitor sobre a autoimagem que o paciente passa a ter de si, sentindo-se vulnerável e isolando-se tanto do convívio familiar quanto social, para isso o enfermeiro deve adotar medidas de segurança para prestar assistência deixando-os assim mais confortáveis e confiantes.

A abordagem do trabalho tem como objetivo geral avaliar a autoimagem dospacientes ostomizados. Tendo como objetivos específicos: apresentar a aceitação da autoestima de pacientes ostomizados; desenvolver estratégias de cuidado de Enfermagem para paciente ostomizados.

Tais objetivos serão apresentados em dois capítulos: o primeiro traz a aceitação da autoestima de pacientes ostomizados. O segundo apresenta o desenvolvimento das estratégias de cuidado de enfermagem para os pacientes ostomizados.

\section{Resultados e Discussão}

\subsection{A aceitação dos pacientes ostomizados}

De acordo com a Organização Mundial de Saúde define qualidade de vida como a percepção do indivíduo de sua posição 
na vida, no contexto da cultura e sistema de valores nos quais ele vive e em relação aos seus objetivos, expectativas,padrões e preocupações, pela importância atribuída ao contexto cultural e valores considerados na avaliação e à relevância das metas, expectativas padrões e preocupações, ou seja, considerar a pessoa com seus objetivos, expectativas, padrões e preocupações, dentro de seu contexto de vida, de cultura e de sistemasde valores (Cesaretti, Santos \& Vianna, 2010).

Refletindo sobre esta definição, e tendo em vista todas as implicações físicas,psicológicas e sociais que a construção de uma colostomia acarreta, pode-se perceber que a pessoa com estoma necessita gerenciar uma multiplicidade de transformações em sua vida e na de sua família de modo a alcançar melhor qualidadede vida (Cesaretti, Santos \& Vianna, 2010).

O conceito de autoestima é definido como a percepção do indivíduo sobre seu próprio valor, e essa percepção é derivada da sua própria imagem corporal, daaceitação do ser pela sociedade, do seu bem-estar físico e emocional e da sua capacidade de adaptação. Depois da confecção do estoma, enfatiza-se a importância de profissionais habilitados, em especial do enfermeiro, em esclareceras dúvidas e auxiliar pessoas com ostomia no processo de adaptação (Melo, 2019).

A imagem corporal está intimamente ligada à autoestima, autoimagem, autoconceito, conceito corporal e esquema corporal, componentes importantes de sua identidade. Desta forma, o paciente ostomizado pode apresentar comportamentos de alienação do seu corpo por sentir-se diferente após a cirurgia, provocando um menor respeito e confiança por si próprio. Não é incomum o choqueprovocado pela $1^{\mathrm{a}}$ observação de sua condição após a cirurgia, causando-lhe, muitas vezes um desgosto assustador (Wall \& Santos, 2017).

Os portadores de ostomia apresentam dificuldades mesmo adaptados, principalmente nos aspectos estéticos e pela insegurança que esse procedimento provoca em alguns, pelo medo de vazamentos, flatulências e de causar incômodosnas pessoas ao seu redor. Ressalta-se que compete, ao enfermeiro, as intervenções exclusivas nos períodos pré, trans e pós-operatórios, visando à melhoradaptação à condição de ostomizado, promovendo o enfrentamento para melhor qualidade de vida do usuário, exigindo cuidado contínuo e prolongado dos serviçosde saúde (Aguiar, 2019).

Apesar de malformações congênitas serem também causas para a ocorrência de estomas, e o paciente convive por toda a vida com o problema, na grande maioria dos casos uma pessoa se torna um paciente ostomizado em um momento posterior, na fase adulta ou na adolescência. Tornar-se um ostomizado éuma situação que causa grandes mudanças na vida do paciente, de sua família e até de amigos (Oliveira \& Oliveira, 2017).

O ostomizado defronta-se com a multiplicidade de alterações de ordem física,psicológica, espiritual, social e sexual, o que pode gerar limitações significativas emseu cotidiano. A saúde, em sua visão holística, enfoca a necessidade de atenção do paciente ostomizado voltada não só para sua nova situação de saúde, mas também para a representação desse estoma para a sociedade, porque para o paciente é muito difícil encarar o preconceito vindo das pessoas que não possuempreparo para conviver com o ostomizado (Couto, 2018).

As alterações da imagem corporal são determinantes na qualidade de vida do paciente nas diversas fases da sua reabilitação, a maioria dos ostomizados sentem-se mutilados após passarem pela cirurgia, e vivem em um período de luto com o próprio corpo (Couto, 2018).

A presença de uma ostomia de eliminação intestinal, com perda de controle da eliminação de fezes e gases, pode conduzira a pessoa ao isolamento psicossocial, à alteração da identidade e autoestima, a sentimentos de desprestígiosocial e de incapacidade na gestão da situação, constituindo-se assim como fatoresinibidores neste processo de transição (Pinho, 2018).

As principais reações e sentimentos causados pela ostomia, destacam-se: vergonha, constrangimento, medo diante da nova situação, mal-estar com odor, limitação e discriminação. Por não ter uma bolsa adequada, a pele fica machucada,assada, dura e ferida. A bolsa descola e as fezes caem, passa constrangimentos na rua e em casa. Assim, não podem sair de casa por não ter segurança, a vida sexual também fica difícil por causa da bolsa (Souza, 2016).

A frequente participação do (a) companheiro (a) e familiares no grupo, o quedemonstra a importância do apoio e suporte 
da família neste processo. Evidencia- se que a presença dos mesmos, pode potenciar o autocuidado do usuário e aumentar sua capacidade de enfrentamento diante da doença, tornando-os assim protagonistas no cuidado (Jkupske, Bisognin \& Krug, 2018).

Um dos fatores mais afetados na população ostomizada é a autoestima, queestá intimamente ligada à imagem que o indivíduo tem de si mesmo. Essa pode serconsiderada como um comportamento positivo ou negativo para um propósito peculiar de cada indivíduo, e ainda pode ser classificada em dois extremos em diferentes fases do ciclo da vida (Souza, 2015).

Para pacientes portadores de ostomia, o desconforto ou incômodo, namaioria das vezes, está relacionado à falta de orientação sobre como usar a bolsa e sobre o autocuidado, além da falta de apoio emocional. Sendo este um dos fatoresque contribuem para a boa adaptação ou não do ostomizado. O suporte emocionaldeve ser dado pela família, amigos e, sobretudo pelo enfermeiro (Souza, 2016).

A presença de uma ostomia de eliminação intestinal é um importante desafiopara os profissionais de saúde, para os doentes e para aqueles que com o doente coabitam. A intervenção específica e sistemática de Enfermagem influencia positivamente o percurso de adaptação à circunstância de viver com uma ostomia (Santos, Fontes \& Nogueira, 2017).

O conhecimento dos níveis de autoestima e qualidade de vida relacionada àsaúde em ostomizados, assim como as mudanças provocadas no seu cotidiano, fornece subsídios para o planejamento da assistência dos profissionais envolvidos no cuidado, em especial o enfermeiro. O conhecimento do profissional enfermeiro possibilita o desenvolvimento de estratégias de intervenção que minimizem os transtornos decorrentes da ostomização e, ainda, a possibilidade de capacitar profissionais envolvidos na assistência (Ferreira, 2017).

Neste sentido, os desafios impostos por esta nova condição poderão ser facilitados com a existência de instrumentos teoricamente sólidos e clinicamente relevantes, que permitam ao enfermeiro orientar e avaliar a prestação de cuidadosde saúde de forma mais completa, rigorosa e ajustada (Pinto, 2016).

O papel dos profissionais de enfermagem é fundamental para promover o autocuidado destes pacientes, nesse sentido, a figura do enfermeiro especialista em estomaterapia, como modelo de Prática Avançada em Enfermagem, é uma alternativa eficaz para desenvolver tanto este tipo de recursos cognitivos- comportamentais como para promover a autonomia dos pacientes em tudo relacionado ao cuidado do estoma (Montoro, 2016).

\subsection{Estratégias de cuidados da equipe de enfermagem com pacientes ostomizados}

O papel da enfermagem é importante nos cuidados do paciente ostomizado,pois o enfermeiro é o profissional capacitado a prestar a devida assistência para proporcionar uma melhor integração do paciente a sociedade. Portanto, devido ao seu conhecimento, os enfermeiros são os profissionais qualificados a conceder auxílio na luta contra as dificuldades enfrentadas (Ribeiro et al., 2016).

O enfermeiro que é capacitado, treinado e possui habilidade para oferecer oscuidados aos pacientes ostomizados é chamado de estomaterapeuta. Esta formação é regulamentada pela International Association for Enterostomal Therapy(Gemelli \& Zago, 2014).

O enfermeiro deve possuir condições de oferecer aos pacientes um cuidado que tenha possua como objetivo a integralidade da atenção em saúde. O aumento de pacientes ostomizados tem crescido mundialmente e isso representa um sério desafio para o sistema de saúde (Bellato \& Castro, 2015).

O profissional de enfermagem quando está frente a um paciente com colostomia deve atuar na educação do mesmo e de sua família. Orientando de acordo ao tipo de alimentação, higiene, direcionando desta forma aoautocuidado realizado pelo ostomizado, proporcionando a evolução da adaptaçãoe o retorno as atividades cotidianas (Menezes, 2013).

Porém, é preciso que se tenha um tempo adequado para que o paciente ostomizado enfrente a sua nova condição de vida e passe a entender a necessidadede auto cuidar-se. $\mathrm{O}$ autocuidado é o conjunto de ações que o ser humano desenvolve consciente 
e deliberadamente, em seu benefício, promovendo o bem- estar e a saúde (Violin, 2015).

O comportamento não assertivo, resultado como específico do diagnóstico, expõe a concepção negativa proveniente da ostomia e as transformações instituídas por ela. As dificuldades enfrentadas pelas pessoas com ostomia ocasionam noções equivocadas.

Diante do papel fundamental do enfermeiro no processo de recuperação da autoestima e adaptação da pessoa com ostomia, o processo de enfermagem é percebido pelos enfermeiros como passo decisório, capaz de fornecer subsídios para prestar assistência de forma integral e individualizada, de acordo com as necessidades de cada indivíduo (Melo, 2019).

Ao conhecer a quantidade e o perfil desses pacientes nesta área de abrangência, os profissionais e os gestores de saúde poderão refletir quanto à assistência prestada, além de possibilitar o planejamento e a implementação de ações de modo a proporcionar uma melhor qualidade de vida a essas pessoas, favorecendo a promoção e o aprimoramento da relação profissionalpaciente (Ecco, 2018).

Considera-se que o apoio institucional e o apoio social são de grande importância para a qualidade de vida desses sujeitos. Várias atividades são preconizadas no sentindo de favorecer o enfretamento desta condição, como a consulta de enfermagem, o diálogo familiar e a troca de experiências entre os pacientes (Ricardo, Santos \& Palermo, 2018).

A equipe de enfermagem necessita de capacitação e treinamento sobre a ostomização para orientar sobre os cuidados que o paciente deverá adotar, com humanização, permitindo que o mesmo esclareça suas dúvidas e expresse seus sentimentos. A família e os amigos são essenciais na recuperação e na manutençãoda vida dos pacientes com ostomia, sendo indispensável haver compreensão emm seu dia a dia, para amenizar seu sofrimento e encontrar novos caminhos (Wall \& Santos, 2017).

Portanto, o suporte emocional, passível de ser compartilhado por toda a equipe e a identificação e desenvolvimento de mecanismos de enfrentamento que podem e devem ser "trabalhados" com o paciente, já nesta etapa, são cuidados importantíssimos neste momento (Freedman, 2012).

\section{Considerações Finais}

Diante dos estudos analisados e do que foi discutido, conclui-se que ocorremmudanças significativas no modo de vida e exigem a busca de diferentes estratégiasde enfrentamento das dificuldades.

O paciente ostomizado, após a cirurgia, poderá vivenciar sentimentos negativos derivados não só da formação do estoma, mas também resultantes da causa da sua origem, como no caso do câncer. A pessoa ostomizada experiencia sentimentos que vão desde revolta a depressão.

Sendo assim, é essencial que os profissionais da área da saúde estejam atentos às reações destas pessoas, observando todas as especificidades na prestação de cuidados de saúde, podendo a reação e o comportamento manifestadovariar ao longo do tempo e de pessoa para pessoa.

Cabe a equipe de enfermagem contribuir de forma efetiva e humana,elaborando um plano assistencial individualizado e sistematizado, durante todo o período perioperatório, ou seja, nos períodos pré, trans e pós-operatórios. Envolveorientação ao paciente sobre o procedimento cirúrgico, as mudanças em sua rotina,horários e tipos de alimentação, ensinar o auto cuidado, com a higienização, troca de bolsa de colostomia, tipo de drenagem e prevenção de lesões periestomais, alémde cuidados técnicos específicos especializados.

Torna-se assim indispensável o apoio encontrado na família, em pessoas expressivas, mas também na base de atendimento profissional, a qual é essencial para uma reabilitação mais rápida e eficaz para uma boa adaptação da pessoa à suanova condição, à condição de pessoa ostomizada.

Acreditamos que todas as intervenções do enfermeiro apresentadas neste estudo, desde o pré-operatório até o pósoperatório contribuem para um atendimentode enfermagem de qualidade frente aos pacientes ostomizados. 
Research, Society and Development, v. 10, n. 11, e410101119956, 2021

(CC BY 4.0) | ISSN 2525-3409 | DOI: http://dx.doi.org/10.33448/rsd-v10i11.19956

Sendo assim, sugerimos a realização de trabalhos futuros, a fim de proporcionar maior articulação da rede de cuidado em saúde, integrando os serviços de saúde mental, favorecendo desta forma a construção das redes sociais e a integralidade do cuidado e apoio ao paciente.

\section{Referências}

Aguiar, F. A. S., et al. (2019). Colostomia E Autocuidado: Significados Por Pacientes Ostomizados. Revista de enfermagem UFPE on line, Recife. 13(1), 105110.

Alves, E. R., et al. (2015). O acolhimento num serviço de saúde entendido como uma redede conversações. Instituto de Medicina Social, Universidade do Estado do Rio de Janeiro/ABRASCO.

Batista, M. R. F. F., et al. (2011). Autoimagem de clientes com colostomia em relação à bolsa coletora. Revista Brasileira de Enfermagem, 64(6), $1043-1047$.

Bellato. R., \& Castro, P. (2015). A condição crônica ostomia e as repercussões que traz para a vida da pessoa e sua família. Cienc.Cuid.Saúde.

Carvalho, S. O. R. M., et al. (2015). “Com um pouco de cuidado a gente vai em frente”:vivências de pessoas com estomia. Texto \& Contexto Enfermagem.

Couto, D., et al. (2018). Assistência De Enfermagem Ao Paciente Ostomizado BaseadoNa Teoria De Dorothea Orem. Brazilian Journal of Surgery and Clinical Research - BJSCR, Minas Gerais. 22(1), 55-58.

Ecco, L., et al. (2018). Perfil de pacientes colostomizados na Associação dos Ostomizados do Rio Grande do Norte. ESTIMA, Braz. J. Enterostomal Ther, São Paulo. 16(0518), 1-8

Ferreira, E. C., et al. (2017). Autoestima e qualidade de vida relacionada à saúde deostomizados. Revista Brasileira de Enfermagem. 70(2), 288-95.

Freedman, R. (2012). Meu corpo, meu espelho: aprendendo a conviver com seu corpo, aaceitar seu visual e a gostar cada vez mais de você. Rosa dos Tempos, Rio de Janeiro - RJ.

Gemelli, L. M. G., \& Zago, M. M. F. (2014). A interpretação do cuidado com o ostomizadona visão do enfermeiro: um estudo de caso. Rev Latino-americana de Enfermagem.

Jkupske, J. W., Bisognin, E., \& Krug, M. M. (2018). Cuidado Ao Usuário Ostomizado: Uma Abordagem Multidisciplinar. Revista Biomotriz, Cruz Alta. 12(3), $78-85$.

Mazalli, E. M. R. (2014). Compartilhando o cuidado da pessoa ostomizada, anais do $2^{\circ}$ Congresso Brasileiro de Extensão. Universitária Belo Horizonte.

Melo, M. D. M., et al. (2019). Diagnóstico de enfermagem baixa autoestima situacional empessoas com estomia: estudo de acurácia diagnóstica. Revista Escola de Enfermagem, 53 (03514), 1-8.

Mendes, K. D. S., et al. (2010). Revisão integrativa: Método de pesquisa para a incorporação de evidências na saúde e na enfermagem.

Menezes, L. C. G., et al. (2013). Prática de autocuidado de ostomizados: contribuições dateoria deorem. Revista Mineira de Enfermagem.

Montoro, C. H., et al. (2016). Vivências e enfrentamento diante da alteração da imagemcorporal em pessoas com estomas digestivos. Revista Latino-Americana de Enfermagem. 24(2840), 1-9.

Oliveira, R. A., \& Oliveira, A. M. L. (2017). Pacientes Ostomizados Em Tratamento No Poliambulatório De Feridas De Foz Do Iguaçu. Br. J. Ed. Tec. Soc. $10(4), 307-317$

Pinho, J., et al. (2018). A Pessoa Com Ostomia De Eliminação Intestinal: Representação Social Dos Enfermeiros. Revista de Investigação \& Inovação em Saúde. $1(2), 23-36$

Pinto, I. E. S., et al. (2016). Propriedades Psicométricas do Formulário Desenvolvimento da Competência de Autocuidado da Pessoa com Ostomia de Eliminação Intestinal. Revista de Enfermagem Referência. 8, 75-84.

Riveiro, R. V. L., Oliveira, A. C., Viana, L. V. M., Pinto, A. P., Carvalho, M. L., \& Elias, C. M. V. (2016). Adaptação social do paciente colostomizado: desafios na assistência de enfermagem. Revista Interdisciplinar.

Ricardo, E. V., Santos, C. M., \& Palermo, T. A. C. (2018). Imagem Corporal E Autoestima Entre Pacientes Com Ostomias Intestinais. Revista Perspectivas Online: Biológicas \& Saúde. 8(28), 71-80.

Violin, D. G. F., et al. (2015). Percepção de pacientes ostomizados sobre os cuidados desaúde recebidos. Rev. Latino-Americana de Enfermagem. 25(1), 1-8. 